\title{
Riesgos de la implementación de los conceptos de intervención humanitaria y la responsabilidad de proteger*
}

\author{
Risks of the implementation of the humanitarian intervention concepts and \\ the responsibility to protect
}

\begin{abstract}
María-Carolina Rendón-Arenas ${ }^{* *}$
*Investigación realizada a través del grupo de Investigación de Estudios Políticos de la Universidad Pontificia Bolivariana.

**Politóloga, Magíster en Estudios Políticos. Actualmente se desempeña como Subsecretaria de Planeación Educativa de la Secretaría de Educación de Medellín. Correo electrónico: carorendon40@hotmail.com Medellín, Colombia.
\end{abstract}

Fecha de recepción: Marzo de 2019

Fecha de aprobación: julio de 2019

\begin{abstract}
Para citar este artículo / To reference this article Rendón-Arenas, M.C (2019) Riesgos de la implememntación de los conceptos de intervención humanitaria y la responsabilidad de proteger.Inciso, 21; 70-83.
\end{abstract}

DOI: http://dx.doi.org/10.18634/incj.21v.1i.927

\section{Resumen}

Luego de 1945, el orden internacional formal liderado por la Organización de las Naciones Unidas modificó un asunto sustancial del orden internacional westfaliano: a partir de entonces, los Estados soberanos no tienen el derecho legítimo a hacer la guerra, salvo en contadas excepciones. El presente trabajo pretende analizar La justificación de las guerras que se han desarrollado bajo el amparo de una de estas excepciones a la prohibición expresa de la guerra: las intervenciones humanitarias y las intervenciones humanitarias hechas desde la noción de la responsabilidad de proteger.

El trabajo está dividido en dos partes: en un primer momento ofrece una caracterización teórica de la diferencia existente entre la responsabilidad de proteger y las intervenciones humanitarias, mientras que en un segundo momento propone un análisis crítico con respecto a las intervenciones humanitarias y las intervenciones humanitarias hechas desde el concepto de la responsabilidad de proteger.

Palabras clave: estado, guerra, relaciones internacionales, derecho internacional, defensa 


\begin{abstract}
After 1945, the formal international order led by the United Nations Organization modified a substantial issue of the Westphalian international order: thereafter, sovereign states have no legitimate right to make war, except in a few exceptions. The present work tries to analyze the justification of the wars that have developed under the protection of one of these exceptions to the express prohibition of war: humanitarian interventions, and humanitarian interventions made from the notion of the responsibility to protect.

This work is divided into two parts: first, it offers a theoretical characterization of the difference between the responsibility to protect, and humanitarian interventions; second, it proposes a critical analysis regarding humanitarian interventions, and humanitarian interventions made from the concept of the responsibility to protect.
\end{abstract}

Keywords: State, war, international relations, international law, defense

\title{
INTRODUCCIÓN
}

Tras el fin de la guerra de los 30 años, que se formalizó a través de un conjunto de tratados firmados en las ciudades centro europeas de Münster y Osnabrück y que se conocen popularmente como la Paz de Westfalia, emergió un orden internacional caracterizado por el creciente protagonismo de los Estados soberanos que prescindieron del reconocimiento de la Iglesia como organismo rector de las relaciones internacionales y, en consecuencia, pasaron a reconocerse en un plano de igualdad formal, para lo cual se crearon cuerpos burocráticos encargados de la diplomacia (Kissinger, 2016, pp. 35-38). Uno de los aspectos en los que los Estados soberanos se reconocieron como iguales fue en el derecho a hacer la guerra que en la modernidad pasó a ser un medio para la consecución de fines políticos, como queda expuesto en el trabajo de Karl Von Clausewitz (2005).

Tres siglos después, cuando finalizaba la segunda guerra mundial se concretó la estructura jurídica de la Organización de las Naciones Unidas (ONU) a través de una Carta que estipula, desde su primera consideración, la voluntad de "preservar a las generaciones venideras del flagelo de la guerra que dos veces durante nuestra vida ha infligido a la Humanidad sufrimientos indecibles". En dicha carta, entonces, queda clara la prohibición formal de la guerra como mecanismo de resolución de conflictos en el sistema internacional, lo que estuvo acompañado de la decisión política de ofrecer mecanismos de cooperación entre los Estados, así como de establecer un catálogo de mecanismos institucionales para el arreglo pacífico de controversias.

No obstante, en el marco de la ONU se establecieron, también, dos casos excepcionales en los que puede recurrirse al uso de la fuerza, o lo que es lo mismo, en los que la guerra estaría permitida: cuando se hace por disposición del Consejo de Seguridad o cuando pueden invocarse los elementos constitutivos de la legítima defensa. Así mismo, por fuera de estos casos excepcionales en los que la institucionalidad de la ONU permite recurrir al uso de la fuerza, autores como Ximena Fuentes (2014) ponen de manifiesto que algunos Estados han alegado la necesidad de hacer uso de la violencia sin necesidad de sobrepasar los límites formales. La legítima defensa preventiva y la intervención humanitaria. 
Tabla 1. Excepciones que permiten el ejercicio de la fuerza en el plano internacional.

\begin{tabular}{|c|c|c|}
\hline Mecanismo & Descripción & $\begin{array}{c}\text { ¿Se encuentra } \\
\text { en la Carta de } \\
\text { San Francisco } \\
\text { de 1945? }\end{array}$ \\
\hline $\begin{array}{l}\text { Por disposición } \\
\text { del Consejo de } \\
\text { Seguridad }\end{array}$ & $\begin{array}{l}\text { Este organismo fue creado a través de la misma Carta de las Naciones Uni- } \\
\text { das (1945) y, a través de este, como señala Paul Kennedy (2007) en el texto } \\
\text { El Parlamento de la Humanidad, "las grandes potencias asumieron la máxi- } \\
\text { ma responsabilidad: la de decidir sobre la guerra y la paz" (p. 84), de con- } \\
\text { formidad con lo dispuesto en los artículos } 24 \text { (Numeral 1) y } 42 \text { de la Carta } \\
\text { Fundacional de la ONU (1945) }\end{array}$ & SI \\
\hline Legítima defensa & $\begin{array}{l}\text { La Carta, en el Artículo 51, enfatiza en el hecho de que ninguna de las dispo- } \\
\text { siciones que apuntan al mantenimiento de la paz y la seguridad internacio- } \\
\text { nal podrá menoscabar "el derecho inmanente de legítima defensa, individual } \\
\text { o colectiva, en caso de ataque armado contra un Miembro de las Naciones } \\
\text { Unidas". Lo anterior, sin embargo, no pude dar lugar a acciones sostenidas } \\
\text { indefinidamente en el tiempo sino "hasta tanto el Consejo de Seguridad haya } \\
\text { tomado las medidas necesarias para mantener la paz y seguridad interna- } \\
\text { cionales", razón por la cual, cualquier Estado que invoque el derecho a la } \\
\text { legítima defensa deberá informar al Consejo de Seguridad. }\end{array}$ & Si \\
\hline $\begin{array}{l}\text { Legítima defensa } \\
\text { preventiva }\end{array}$ & $\begin{array}{l}\text { Como se mencionó, el Artículo } 51 \text { de la Carta de las Naciones Unidas permite } \\
\text { expresamente la legítima defensa en los casos en los que se presenta un } \\
\text { ataque armado en contra de alguno de los Estados miembros de la ONU. Sin } \\
\text { embargo, ha surgido el debate alrededor de la posibilidad de incurrir en el } \\
\text { ejercicio de la fuerza cuando existe la amenaza razonable de que se produz- } \\
\text { ca dicho ataque. Tener claridad sobre este aspecto es importante toda vez } \\
\text { que en una era de proliferación de armas nucleares, "esperar a que otro Es- } \\
\text { tado tire la primera piedra puede simplemente dejar en la total indefensión } \\
\text { y llevar a la destrucción total del Estado atacado" (Fuentes, } 2014, \text { p. } 263)^{1} \text {. }\end{array}$ & No \\
\hline $\begin{array}{l}\text { Intervención hu- } \\
\text { manitaria }\end{array}$ & $\begin{array}{l}\text { Algunos actores políticos justifican que en los casos en los que hay violacio- } \\
\text { nes de derechos humanos puede hacerse uso de la violencia bajo el amparo } \\
\text { de dos razones rescatadas por Fuentes (2014): la primera de ellas, en tanto } \\
\text { que no se transgrede lo dispuesto por el artículo } 2.4 \text { de la Carta de las Na- } \\
\text { ciones Unidas }{ }^{2} \text { y, la segunda de ellas, "porque ha surgido una nueva norma } \\
\text { consuetudinaria que la permite" (p. 264). }\end{array}$ & No \\
\hline
\end{tabular}

Fuente: Elaboración propia basado en Carta de San Francisco de la Organización de las Naciones Unidas (1945), Kannedy (2007), Fuentes (2014)

Adicional a lo anterior, durante la Cumbre Mundial del 2005, Kofi Annan, entonces secretario general de la ONU, lideró la formulación de otro mecanismo de excepción al uso de la fuerza en el ámbito internacional mediante un concepto propio del derecho internacional público que invoca la responsabilidad que tiene la comunidad de Estados en el ámbito internacional de proteger a los pobladores de un territorio específico de ser víctimas de crímenes de lesa humanidad, cuando el Estado garante de su seguridad se encuentre en incapacidad de hacerlo. Dicho propósito se encuentra consignado en el Documento Final de la Cumbre Mundial de la Asamblea General y desarrollado en los párrafos 138 y 139 del mismo (Organización de las Naciones Unidas, 2005) 
Lo anterior, es un tema de importancia capital dentro de nichos disciplinares como la Ciencia Política, el Derecho, las Relaciones Internacionales, entre otras, toda vez que las intervenciones humanitarias y la responsabilidad de proteger han sido un punto importante de la agenda internacional de las últimas décadas y, de la misma manera, ha sido objeto de discusión en el marco de coyunturas críticas que suponen casos como los de Siria y Venezuela, entre otros.

Este trabajo se presenta como un documento reflexivo resultado de una investigación realizada con anterioridad, durante del proceso de confección del trabajo para optar al título de Magíster en Estudios Políticos de la Universidad Pontificia Bolivariana, en la que se identificaron voces críticas como las de Danilo Zolo, Franz Hinkelammert, Emmanuel Guerisoli, entre otros autores que inspiraron la realización de este texto.

Los asuntos planteados con anterioridad sobre la responsabilidad de proteger y las intervenciones humanitarias se desarrollarán en dos capítulos: el primero de ellos presenta una caracterización teórica de ambos conceptos, aspecto que es empalmado con las voces críticas que, desde la academia, se han tejido alrededor de los mismos. El segundo de ellos ofrece una reflexión personal sobre el impacto y las diferencias existentes entre las intervenciones humanitarias y la responsabilidad de proteger.

Lo anterior, será abordado desde un enfoque institucionalista, el cual permite confrontar la forma, organización y funcionamiento de las instituciones políticas, así como su impacto en la práctica política (Cardona \& Mejía, s.f., p. 2), elementos que son identificables en las acciones, decisiones y omisiones que tienen lugar en el marco del orden internacional formal de las Naciones Unidas y en el marco del sistema político, informal, es decir, en el que se desenvuelve la competencia por el poder entre distintos actores.

\section{UNA DIFERENCIACIÓN CLAVE: LA RESPONSABILIDAD DE PROTEGER Y LA INTERVENCIÓN HUMANITARIA}

Con el objeto de comprender de mejor manera el trabajo planteado es necesario definir y diferenciar los conceptos de la responsabilidad de proteger y la intervención humanitaria, soportada en la noción de humanitarismo. En la recta final de este capítulo, se ofrece una caracterización de las voces críticas con respecto a la noción de la responsabilidad de proteger.

\section{LA RESPONSABILIDAD DE PROTEGER}

A partir de los conflictos desarrollados en Ruanda, Somalia, Kosovo y Bosnia, en la década de los noventa, se hizo notoria una debilidad propia de la reacción que tuvo la comunidad internacional. Lo anterior, toda vez que no existía claridad sobre las condiciones bajo las cuales se debía desarrollar una intervención humanitaria cuando una población se encontrara en situación de vulnerabilidad (Organización de las Naciones Unidas, 2012). Por lo anterior, el entonces Secretario General de la ONU, Kofi Annan abrió el debate sobre la intervención humanitaria a raíz de estos conflictos, mencionando que:

Si la intervención humanitaria es, en realidad, un ataque inaceptable a la soberanía, ¿cómo deberíamos responder a situaciones como las de Ruanda o Srebrenica y a las violaciones graves y sistemáticas de los derechos humanos que transgreden todos los principios de nuestra humanidad común? (Naciones Unidas, 2012). 
Como respuesta a la pregunta planteada por Annan, la Comisión Internacional sobre Intervención y Soberanía de los Estados, la cual se estableció por el gobierno de Canadá, utiliza por primera vez el concepto responsabilidad de proteger en uno de sus informes (2001) en donde concluían que:

la soberanía no solo daba al Estado el derecho de "controlar" sus asuntos, sino que le confería la "responsabilidad" primordial de proteger a la población dentro de sus fronteras. También proponía que cuando un Estado no protegiese a su población, ya fuera por falta de capacidad o de voluntad, la comunidad internacional en general asumiría esa responsabilidad (Naciones Unidas, 2012).

Las Naciones Unidas acogieron el concepto de la responsabilidad de proteger en 2004 fundamentándola en la responsabilidad colectiva internacional a través del Consejo de Seguridad, organismo al cual le fue asignada una autorización para intervenir de manera militar, en caso de ser necesario y como último recurso ( último recurso no debe entenderse como el último medio que puede utilizarse cuando se han agotado todos los otros; por el contrario hace referencia a la opción más viable para lograr un objetivo (Bellamy, 2009)) frente a violaciones graves del derecho internacional humanitario en donde los gobiernos soberanos no demostraran capacidad para evitarlo o, simplemente, que no lo quisieran evitar (Naciones Unidas, 2012). Lo anterior implica que estas intervenciones soportadas en la responsabilidad de proteger cobijan, al igual que el derecho internacional humanitario, tanto las guerras internacionales, como los escenarios de conflicto armado interno.

La responsabilidad de proteger contiene dos principios básicos bajo los cuales se fundamenta: el primero hace referencia a la responsabilidad que tiene cada Estado soberano de proteger a su población de un daño grave, mientras que el segundo concierne a la responsabilidad internacional que tienen los Estados cuando se realiza un daño a una población como resultado de una guerra civil, una represión o un acto que ponga en situación de vulnerabilidad a un grupo determinado. Es necesario recordar que esta responsabilidad que adquieren los Estados se sobrepone al principio de no intervención que surgió de manera posterior a la Paz de Westfalia (ICISS, 2001).

Dentro de la responsabilidad de proteger se encuentran articulados elementos que le dan sentido al concepto, como lo son: primero, la responsabilidad de prevenir, segundo, la responsabilidad de reaccionar y tercero, la responsabilidad de reconstruir.

En primer lugar, la responsabilidad de prevenir busca, principalmente, que no existan causas directas que permitan que se desarrollen conflictos que puedan poner en peligro a la población; en segundo lugar, la responsabilidad de reaccionar busca que, en los momentos que sea necesario, intervenir se realice efectivamente, ya sea de forma coercitiva por medio de sanciones económicas o por medios militares; mientras que, en tercer lugar, la responsabilidad de reconstruir busca que se brinde, en la etapa posterior a una intervención militar, ayuda para la reconstrucción, recuperación y reconciliación de la población afectada (ICISS, 2001).

A comienzos del siglo XXI, Francis Deng desarrolló el concepto de soberanía como responsabilidad con el cual busca "sobrepasar la utilización de ese atributo estatal cuando se ejerce como 
obstáculo, auténtica barricada para obstruir la función de la asistencia internacional a los ciudadanos de un Estado perpetrador de crímenes atroces o es incapaz de impedir que se cometan" (Citado en Méndez, 2016, p. 6). Por lo tanto, cuando un Estado cumple con su responsabilidad soberana logra integrarse completamente al sistema internacional, de lo contario, cuando no lo hace se puede recurrir a una intervención con el fin de proteger a la población en cuestión.

Este concepto sugiere una reinterpretación del concepto de soberanía y el principio de la no intervención. La soberanía clásica se ha visto desde la Paz De Westfalia como la capacidad de autodeterminación de los Estados y como el poder independiente de cualquier injerencia externa. La intervención se ha visto tradicionalmente como un atentado contra la voluntad de una nación. Dicha percepción comenzó a cambiar desde el derecho internacional cuando se formó la Liga de las Naciones posterior a la primera guerra mundial. En esta coyuntura no se pasó abiertamente a legitimar las intervenciones humanitarias, más si se abrió una nueva etapa en donde los Estados estaban atados bajo una serie de principios morales que debían ser cumplidos por todos para poder obtener una armonía más fuerte en las relaciones internacionales. En caso de que algún Estado violara algún principio de convivencia pacífica con los demás miembros de la Liga, se condenaría la acción dentro de la naciente sociedad. A partir de aquí los países se la pensarían dos veces para atacar otro país o atentar contra su propia población. La defensa de la población de cada país desde la mirada de otras naciones se reforzó con la aparición de la Declaración Universal de los Derechos Humanos de 1948. Los seres humanos, luego de esto, serían mirados con una condición de paridad tal, que ningún estado podría cometer atrocidades contra su población sin sufrir una denuncia internacional de otros países y de la opinión pública mundial. El momento histórico donde más se tuvieron en cuenta las premisas anteriores fue luego del fin de la guerra fría. En la nueva dinámica del mundo globalizado se esbozó el espíritu del posterior concepto de la responsabilidad de proteger a través de diversas intervenciones humanitarias, como las de Bosnia-Herzegovina, Ruanda, Somalia y Kosovo. En este cambio axiológico,

no se trata de lograr un mundo seguro para las grandes potencias ni de pisotear los derechos soberanos de las más pequeñas, sino de ofrecer protección práctica a las personas corrientes cuya vida corre peligro porque sus Estados no quieren o no pueden protegerlas (ICISS,2001, p. 27)

Los Derechos Humanos, que cada vez cobran mayor fuerza, son generalmente aceptados por la comunidad internacional, y son los principales forjadores de un cambio de perspectiva.

Más que un derecho a intervenir, los Estados tienen una obligación erga omnes de tomar, cuanto antes, todas las medidas a su alcance para prevenir o poner fin a las violaciones graves y masivas a los derechos humanos (Arredondo, 2001, p. 47.) 


\section{Humanitarismo e INTERVEnción hUMANITARIA}

De la mano del derecho internacional humanitario se encuentra el término que autores como Walzer (2011) denominan humanitarismo. El humanitarismo, según el autor, dependiendo de la interpretación que se le dé, puede tener una connotación positiva o negativa, es decir, el humanitarismo se encuentra en la dicotomía entre si es un acto por parte de un Estado que se realiza por caridad o si por el contrario se aleja de lo voluntario y se convierte en un deber que de manera tácita adquieren los Estados como miembros del sistema internacional.

Frente a un desastre natural o una situación en la que una población se encuentre vulnerada, la ayuda humanitaria parece ser un acto positivo dado que lo que busca es lograr disminuir o finalizar con el sufrimiento de las personas. Sin embargo, esta conducta generosa simplemente responde al deber ser que las personas y los Estados, de una u otra manera, se encuentran ligados a realizar. Por lo tanto, el humanitarismo internacional representa un imperativo que combina el deber de ayudar y el querer hacerlo.

En cuanto a la política del humanitarismo, en la sociedad internacional a pesar de contar con una autoridad responsable o a cargo como las Naciones Unidas, ésta muchas veces no satisface las necesidades que las personas en situación de vulnerabilidad pueden tener por el poder de veto que limita la capacidad de tomar acciones, además de un fuerte sentido de responsabilidad hacia la responsabilidad global o el bienestar en general (Walzer, 2011).

En el caso de la intervención humanitaria, también se puede apreciar este dilema cuando, según Walzer:

Los gobiernos pueden utilizar la fuerza para detener una masacre, como Estados Unidos, Francia y el Reino Unido aseguran estar haciendo en Libia, y como debió hacerse en Ruanda. Podemos pensar que esto es una dádiva para las personas que serán rescatadas, y que se trata de una donación considerable, ya que puede incluir la vida de algunos de los que intervienen. ¿Pero el Estado que interviene actúa de forma caritativa? ¿Acaso detener una masacre no es moralmente necesario? (2011, p. 72-73).

En sentido estricto, la intervención Humanitaria se entiende como el uso de la fuerza armada, de uno varios Estados, u organizaciones internacionales, en contra de otro Estado y sin autorización de éste; cuya finalidad es la de prevenir o poner fin a las violaciones graves y masivas de los DDHH y el DIH; es una respuesta inmediata, a través de una acción armada positiva, respecto a una amenaza humanitaria abrumadora, pues se quiere evitar la perpetración de violaciones masivas y flagrantes del DIH y los DDHH. Esto, para algunos, incluye la implementación previa de medidas que no implican el uso de la fuerza para evitar este tipo de situaciones (sanciones políticas o diplomáticas, medidas económicas, acciones legales o judiciales, entre otras), así como las empleadas para la consolidación de la paz y la reconstrucción, posteriores a la realización de la acción coercitiva (Arredondo, 2001, p. 26-35.) ${ }^{1}$

1. En este sentido, la Comisión Internacional sobre intervención y Soberanía, entiende la intervención como una responsabilidad discriminada en tres tipos: la de prevenir, la de reaccionar y la de reconstruir (ICISS, 2001). 


\section{VOCES CRÍTICAS ALREDEDOR DEL CONCEPTO DE INTERVENCIÓN HUMANITARIA}

Desde una perspectiva crítica y escéptica, la intervención humanitaria es vista como una manera de torpedear el atributo básico de los Estados que se hereda desde el sistema westfaliano clásico: la soberanía. La Carta de creación de la ONU es vista como el mito fundacional de una ola institucional que corroe las prerrogativas de poder de las naciones. Las premisas de acabar las guerras (consideradas la peor atrocidad contra la humanidad) y de promover los derechos humanos han generado, paradójicamente, un sistema internacional poco pacífico y más anárquico. La concepción wilsoniana de "la guerra que le pondrá fin a todas las guerras" está a la orden del día en las relaciones internacionales cuando se alude a las intervenciones humanitarias.

Las objeciones teóricas formuladas al concepto de intervención humanitaria no apuntan a un mismo asunto: dentro de este catálogo académico pueden destacarse posturas que cuestionan dicho mecanismo desde la parte jurídica, desde la parte discursiva y desde el interés nacional estadunidense.

Danilo Zolo (2007) asevera que todos los intentos jurídicos del Siglo XX han tenido la intención de cercenar la posibilidad de la existencia del lus Bello, que como bien se sabe, fue la base de las Relaciones Internacionales desde Westfalia. Por su parte, se han amalgamado fuerzas para establecer un rígido lus contra bellum, que para nada ha logrado su cometido. El autor dice expresamente que:

la proscripción jurídica de la guerra no dio lugar a un sistema normativo unitario, coherente y exhaustivo, capaz de obtener, aunque sea de modo imperfecto y tendencioso, el objetivo declarado, esto es, la pacificación del mundo, esa pacificación que el sistema westfaliano no había logrado garantizar (Zolo, 2007, p.62)

La ausencia de resultados alentadores se debe al trasfondo político del asunto. Quienes con más vehemencia criminalizan la guerra, defienden los derechos humanos y más reivindican la existencia de órganos como la ONU para que se dé la paz mundial, hacen un uso discrecional de la misma guerra y hacen agresiones contra ciertos regímenes (arguyendo la protección de la población civil) cuando ven que éstas pueden repercutir en su posicionamiento en el orden internacional.

El ahorcamiento de la guerra como facultad de los Estados soberanos propio del sistema westfaliano se modifica por una nueva guerra global, en donde sólo tienen el poder de emprenderla las potencias que ganaron la segunda guerra mundial, esto es, los Estados que son miembros permanentes del Consejo de Seguridad.. En ésta guerra global es donde tiene lugar la guerra preventiva o la guerra contra el terrorismo; las potencias están provistas de una inmunidad jurídica que ellas mismas se procuraron con el establecimiento de la ONU.

El problema de las intervenciones humanitarias, desde el entendido de Zolo, es que no hay una entidad imparcial con dientes que pueda hacer un control posterior a las acciones desplegadas en estas. Si una intervención no tuviera una justificación clara y se fuese a sancionar a una potencia por ello, el Consejo de Seguridad, como ejemplo de entidad revisora, pelearía contra 
sí mismo, ya que la potencia que se va a reprender es a su vez parte de los encargados de la punición. Estaríamos entonces ante el absurdo de jueces que se juzgan por crímenes cometidos.

Por su parte, autores como Franz Hinkelammert (1999) destacan que las intervenciones humanitarias son guerras disfrazadas, es decir, guerras convencionales que depuran semánticamente el contenido bélico, cuando en la realidad "lo humano" de la intervención es una arbitrariedad del poderoso que puede realizar la misma y deslindar quienes merecen vivir de los que no, anotando, además, que en este tipo de guerras

La responsabilidad por el aniquilamiento la tiene quien es aniquilado. Quien aniquila, en cambio, tiene el poder e igualmente el honor de respetar los derechos humanos. Es el prócer de los derechos humanos, y la sangre que vierte, lo purifica (Hinkelammert, 1999, p. 20)

Si bien las dos críticas anteriores resaltan las móviles de fuerza y despliegue de poder que están detrás de las intervenciones filantrópicas de las potencias en general, hay una crítica encaminada a desnudar las intervenciones humanitarias como algo de una potestad casi exclusiva de los Estados Unidos y que se impulsó férreamente desde el gobierno de Clinton. La idea de ser la policía mundial por parte de los Estados Unidos está encarnado en un fenómeno que se denomina la Doctrina Clinton:

Tal política, apodada como la doctrina de las "Guerras Humanitarias", fue descrita como la facultad, o mejor el derecho, de los Estados Unidos de intervenir militarmente en otros estados con el fin de acabar con la persecución o los asesinatos en masa de poblaciones civiles por razones étnicas, raciales o religiosas. Clinton definió al genocidio y a los delitos de lesa humanidad como intereses nacionales, permitiendo así, una interferencia directa. Por lo tanto, los Estados Unidos se encontrarían obligados a actuar si tuviesen la oportunidad y estuviesen preparados para terminar con la perpetración de crímenes contra la humanidad en cualquier parte del mundo; como también, para impedir la propagación de aquellos conflictos y sus consecuencias. De esta forma los Estados Unidos asumirían el rol de "policía mundial" obteniendo el legítimo uso de la fuerza. (Guerisoli, 2006, p.1)

Aunque es cierto que la Doctrina Clinton puede tener unos fines anclados a la moral y que propende por salvaguardar vidas inocentes, han surgido críticas, tanto de los actores políticos de derecha e izquierda, que despojan su esencia.

Por una parte, los pensadores realistas y algunos liberales han sustentado que intervenciones tales como las que se hicieron por el Gobierno Clinton en lugares como los Balcanes, Somalia y Haití no son herramientas que redunden en beneficio de los intereses nacionales estadounidenses. Esa labor social sin sentido, a la que se refieren internacionalistas como Michael Mandelbaum, alejan a Estados Unidos de sus relaciones con potencias como Rusia o China. Los norteamericanos deberían centrarse más, bajo esta perspectiva, en resolver problemas internacionales inminentes como el de Irán y Corea del Norte (problemas que son potencialmente destructivos) y no en resolver asuntos internos de países periféricos.

En el otro lado del espectro ideológico, intelectuales como Noam Chomsky criticaron las acciones enmarcadas en la Doctrina Clinton porque vulneraban la soberanía de algunos Estados y porque se abusaba de una fuerza ostensiblemente más poderosa en contra de la debilidad de estos 
mismos Estados. El hecho de querer instaurar democracias a través de la violencia también se ha intentado desenmascarar por parte de esta corriente de pensamiento. (Guerisoli, 2006, p.12)

\section{RefLeXIÓN SOBRE LAS INTERVENCIONES HUMANITARIAS Y LA RESPONSABILIDAD DE PROTEGER}

El nuevo concepto de la responsabilidad de proteger ha sido considerado por muchos autores y actores políticos como la versión más depurada del gran espectro de las intervenciones humanitarias. El nuevo siglo se abrió con un término sofisticado para las nuevas relaciones internacionales. En el centro de discusiones deberían estar los pueblos y las personas, restando protagonismo a los regímenes y los Estados. La división clásica entre conflictos internos y amenazas a la paz y seguridad internacionales no debería ser tan tajante y debería flexibilizarse en nombre de los derechos humanos. El propio Kofi Annan nos dice, reinterpretando la Carta de las Naciones Unidas y justificando el nuevo concepto que

la Carta estaba escrita en nombre de "nosotros, los pueblos", no de "nosotros, los Gobiernos" de las Naciones Unidas. Su objetivo no es solo preservar la paz internacional- por vital que sea la importancia de esto-, sino también "reafirmar la fe en los derechos humanos fundamentales, en la dignidad y el valor de la persona humana". El objetivo de la Carta nunca fue otorgar a los Gobiernos una licencia para negar los derechos humanos o la dignidad humana. La soberanía siempre implicó no solo poder sino también responsabilidad (Annan \& Mousavizadeh, 2012, p. 134)

Si bien se han esgrimido argumentos como el anterior para consolidar el principio de la responsabilidad de proteger, lo cierto es que la realidad ha demostrado con suficiencia la inoperatividad de este y la confusión semántica que esto ha traído consigo para el léxico de las relaciones internacionales y del derecho internacional. No por crear vocablos nuevos se cambia el mundo de lo factual.

Las intervenciones humanitarias han seguido teniendo los mismos vicios con o sin la responsabilidad de proteger. Las tensiones y las arbitrariedades entre los miembros de la comunidad internacional para tratar conflictos internos de Estados que precisan de una intervención no se han modificado luego del 2001, cuando se crea el concepto. Paradójicamente, las intervenciones humanitarias que no han estado amparadas por el concepto de la responsabilidad de proteger mostraron tener en ciertos casos más naturalidad y eficacia que las intervenciones que se han intentado invocar a través de la figura de la misma responsabilidad de proteger.

Sigue habiendo unas constantes que no se han modificado y que deben tenerse en cuenta a la hora de analizar problemáticas humanitarias, aunque también ha habido algunos cambios positivos, y al mismo tiempo, retrocesos. Por un lado, sigue siendo poco claro el criterio de los miembros de la comunidad internacional a la hora de decir cuáles crisis ameritan la intervención y cuáles no. No existe una lista de características que permita tener de antemano una estandarización de los factores objetivos para que se lleven a cabo las intervenciones humanitarias. Estas intervenciones tienen una naturaleza contingente y arbitraria, lo que hace que lo circunstancial guíe la decisión acomodaticia de los posibles intervinientes. Se puede decir que no existe

una coherencia de la comunidad internacional y de sus instituciones a la hora de diagnosticar las situaciones en las que los derechos humanos de la población son vulnerados (...) y posteriormente 
decidir cuáles son los instrumentos y medidas más adecuados para poner fin a esas graves violaciones de los derechos más fundamentales. Igualmente, el consenso entre los distintos estados de la sociedad internacional ha estado en el punto de mira, además de las opiniones y decisiones adoptadas en el seno de la ONU y en organizaciones internacionales regionales (Marrero, 2012, p. 129)

Existen activismos particulares por parte de sectores de la comunidad internacional ante ciertos casos y silencio e inacción frente a otros casos de similar magnitud. Se creyó que, con las fructíferas intervenciones en Timor y Kosovo, en 1999, se habían corregido los errores de pasividad respecto a los casos de Somalia, Bosnia y Ruanda y que, con la repotenciación discursiva de las intervenciones humanitarias a través de la creación del concepto de la responsabilidad de proteger, el mundo estaba incursionando en una era justa e igualitaria con respecto al cuidado de los derechos humanos en situaciones de crisis. Pero la realidad mostró, luego, que estos dos casos exitosos fueron un espejismo y se volvió al mismo estado original de negligencia en lo corrido del Siglo XXI. La situación de Darfur advirtió que seguiría habiendo casos en los que la comunidad internacional no moverían un solo dedo para solucionar injusticias y violaciones de derechos patentes. No tardó mucho tiempo en que la contradicción emergiera de nuevo: en un mismo año dos situaciones parecidas fueron tratadas de manera radicalmente distinta: las mencionadas de Libia y Siria. En ambos casos no se acudió a los mismos instrumentos, no se aplicaron los mismos tiempos y tampoco ha sido similar el alcance de la actuación de las instituciones y potencias de la comunidad internacional.

Si esta situación de la indefinición de los criterios para intervenir es problemática, es más problemática aún que la misma institución que forjó el concepto de la responsabilidad de proteger justifique la idoneidad de la diferenciación frente a los casos. Lo hizo a través de su líder, el Secretario General:

El contexto es importante. La responsabilidad de proteger es un principio universal. Su aplicación, sin embargo, debe respetar las diferencias institucionales y culturales de una región a otra. Cada región llevará a la práctica el principio a su propio ritmo y a su propia manera (...) No podemos aplicar un único patrón rasero o molde a todas las regiones. Los activos y las necesidades varían entre países y entre regiones (Organización de las Naciones Unidas, 2011)

Si la responsabilidad de proteger es un principio serio y "universal", ante situaciones del mismo talante se debería actuar igual, indistintamente de las particularidades del país. No hay violaciones de derechos humanos más relevantes que otras y es absurdo que los casos de unos países dados susciten el apoyo de la comunidad internacional mientras que los casos de otros suscitan desidia.

Muy relacionado con el punto anterior, y que también sigue siendo una constante en la actualidad y de las intervenciones humanitarias clásicas, es que los intereses políticos de los Estados son los que mueven las decisiones de índole moral también. Un Estado no intervendrá ni ayudará a intervenir en otro Estado si ve que su imagen y si poder pueden verse socavados de algún modo. Aparte de esto, el sistema de alianzas y los contactos estratégicos con determinados países vician las decisiones en materia de intervención de las potencias. La premisa es que nunca se intervendría en contra de un régimen aliado, como vemos diáfanamente con Rusia y el caso de 
Siria. Rusia, que no ha hecho intervención humanitaria allí, sólo ha intervenido para perpetuar en el poder a Al Asad.

Hay pues intereses encriptados a la hora de ponderar la idoneidad de una intervención. El Consejo de Seguridad, que es el órgano legitimado para autorizar las intervenciones humanitarias está movido por los intereses mencionados. No hay neutralidad de ningún tipo en el seno de esta institución político-militar. Es bien sabido que la composición del Consejo sepulta de antemano cualquier fin noble o humanitario: China y Rusia, por lo general, vetarán cualquier decisión promovida por Estados Unidos, Reino Unido y Francia y lo harán simplemente por equilibrar el poder de los distintos bloques a escala planetaria. Como menciona Marrero (2012),

la realidad es que el Consejo de Seguridad no piensa actuar de la misma manera en todos los casos en los que la población está siendo masacrada por las autoridades de un estado, sobre todo si las circunstancias políticas y geoestratégicas lo desaconsejan, como ocurre en Siria. (p. 141)

Enfatizando un poco en lo anterior, es posible visualizar que, si bien la paralización del Consejo de Seguridad se ha presentado en las dos etapas analizadas, en la etapa de la responsabilidad de proteger se reivindican más los dictámenes de esta institución, siendo la única entidad que puede tomar legalmente las riendas de una intervención. Dicho asunto es una involución respecto a la época de la intervención humanitaria tradicional, ya que países o instituciones como la OTAN podían antes intervenir con cierto margen de legitimidad sin la aprobación expresa del Consejo. No parece pues que

la nueva figura de la "responsabilidad de proteger" haya supuesto un gran progreso en relación con la denominada «intervención humanitaria", compartiendo ambas los mismos fundamentos. Es más, se podría decir incluso que ha supuesto un cierto retroceso, sobre todo a partir del Informe del Grupo de Alto Nivel, al restringir el recurso a dicha figura en caso de reacción armada a la estricta aprobación del Consejo de Seguridad. Se han recortado, pues, los supuestos excepcionales a los que hacía alusión el Informe de la comisión canadiense, que abría la espita, llegado el caso, a una intervención unilateral siempre y cuando se reunieran los elementos objetivos de la intervención armada y el Consejo de Seguridad no lograra aprobar una resolución al respecto" (Bermejo \& López-Jacoiste, 2013, p. 72)

Por otro lado, existe otra desmejora en cuanto a las intervenciones humanitarias con la nueva figura de la responsabilidad de proteger: el cambio de los fines y los propósitos primarios por otros objetivos. La naturaleza de una intervención de este tipo es proteger a civiles vulnerables y no irse lanza en ristre contra un régimen determinado y deponerlo, como se observó en Libia.

Es alarmante el hecho de que ante un conflicto interno dado en un país se tome partido explícita o implícitamente por una de las partes o facciones y se aluda al discurso de la protección de la población civil. Lo que debería importar a la hora de una intervención humanitaria son las vidas de las personas indistintamente del bando al que pertenezcan.

\section{Conclusión}

La creación del concepto de la responsabilidad de proteger a comienzos del Siglo XXI puede entenderse como una innovación meramente lexical que no necesariamente marcó diferencia, 
en su implementación, con las intervenciones humanitarias. Ambos mecanismos de justificación del uso de la fuerza en el ámbito internacional guardan similitudes en aspectos formales y de fondo en lo que respecta a la inexistencia de criterios objetivos que permitan ponderar qué situaciones ameritan intervención y cuáles no, condición que propicia el doble racero con el que los Estados, e incluso el Consejo de Seguridad de la Organización de las Naciones Unidas, califican algunas vulneraciones de derechos humanos como más o menos graves que otras, dependiendo del interés político que medie.

Quizás la supervivencia de la responsabilidad de proteger dependa exclusivamente de retomar los valores intrínsecos y primigenios de las intervenciones humanitarias clásicas y de acudir a unos mínimos legales y una caracterización objetiva que permitan un abordaje más imparcial y neutral de las dinámicas que aludan al sufrimiento humano derivado de la vulneración de los derechos.

\section{Referencias biblográficas}

Annan, K. \& Mousavizadeh, N. (2012). Intervenciones: una vida en la guerra y en la paz. Barcelona: Taurus

Arrendondo, R. (2012). Intervención humanitaria y responsabilidad de proteger: ¿hacia un nuevo paradigma de protección de los derechos humanos? Buenos Aires: Catálogos.

Bellamy, A. (2009). Guerras justas de Cicerón a Iraq. Madrid: FCE

Bermejo, R. \& López-Jacoiste, M (2013). De la intervención por causas humanitarias a la responsabilidad de proteger. Fundamentos, similitudes y diferencias. Cuadernos de estrategia, Núm. 160, pp. 18-76.

Cardona, P. \& Mejía, J. (s.f.) Enfoques, métodos y técnicas de la investigación política. Facultad de Ciencias Políticas. Universidad Pontificia Bolivariana

Clausewitz, C. (2005) DE LA GUERRA. Madrid: La Esfera De Los libros, S.L.

Guerisoli, E. (2006). La doctrina Clinton: las guerras humanitarias" a. Programa Teoría de Relaciones Internacionales.

Hinkelammert, F. (1999) La inversión de los derechos humanos: el caso de John Locke. Pasos, Núm. 85, pp. 20-35. Recuperado de: http://repositorio.uca.edu.sv/jspui/ bitstream/11674/865/1/La\%20inversi\%C3\%B3n\%20de\%20los\%20derechos\%20 humanos\%20el\%20caso\%20de\%20John\%20Locke\%20\%281\%29.pdf

ICISS. (2001). Responsabilidad de Proteger. Informe de la Comisión Internacional sobre intervención y soberanía de los Estados. Recuperado de: http://www.ceipaz.org/images/ contenido/La\%20responsabilidad\%20de\%20proteger_ESP.pdf 
Kennedy, P. (2007). El Parlamento de la Humanidad. Bogotá: Random House Mondadori, S.A.S.

Kissinger, H. (2016) Orden Mundial. Reflexiones sobre el carácter de los países y el curso de la historia. Bogotá: Penguin Random House Grupo Editorial, S.A.U.

Marrero, I. (2013). La responsabilidad de proteger de la comunidad internacional en los casos de Libia y Siria: análisis comparativo. Relaciones Internacionales. Núm. 22. UAM

Méndez, E. (2016). Responsabilidad de proteger: la ONU en acción. Real Instituto Elcano Royal Institute, España. Recuperado de: http://www.realinstitutoelcano.org/wps/wcm/ connect/efd134004bb105749fbfbf6e9c1af357/DT2-2016-MenendezDelValle-Responsabilidad-de-proteger-ONU-en-accion.pdf?MOD=AJPERES\&CACHEID=efd134004bb$105749 f b f b f 6 e 9 c 1 a f 357$

Organización de las Naciones Unidas (1945). Carta de las Naciones Unidas.

Organización de las Naciones Unidas (2005) Documento Final de la Cumbre Mundial, Asamblea General.

Organización de las Naciones Unidas (2011) La función de los mecanismos regionales y subregionales para hacer efectiva la responsabilidad de proteger, Informe del Secretario General, A765/877-S/2011/393, párrafo 8.

Organización de las Naciones Unidas. (2012). La responsabilidad de proteger. Programa sobre el genocidio de Ruanda y las Naciones Unidas. Recuperado de: http://www.un.org/es/ preventgenocide/rwanda/pdf/bgresponsibility

Walzer, M. (2011). Sobre el humanitarismo. Foreing Affairs Latinoamérca. Vol. 11, Número 4, pp. 72-82. Recuperado de: http://revistafal.com/numeros-anteriores/fal-11-4/

Zolo, D. (2007). La justicia de los vencedores: de Nuremberg a Bagdad. Trotta.

\section{(FoOTNOTES)}

1. Para más claridad sobre lo anterior, puede consultarse el trabajo de Alex Bellamy (2009) en el que se diferencia teóricamente entre la guerra preventiva y la guerra preventiva.

2. El Numeral 4 del Artículo segundo de la Carta de las Naciones Unidas estipula que "Los Miembros de la Organización, en sus relaciones internacionales, se abstendrán de recurrir a la amenaza o al uso de la fuerza contra la integridad territorial o la independencia política de cualquier Estado, o en cualquier otra forma incompatible con los Propósitos de las Naciones Unidas". 